\title{
Educational Tools to Introduce Computer- Aided Drug Design to Students and to the Public at Large
}

\author{
Antoine Daina ${ }^{\text {ał }}$, Marie-Claude Blatter ${ }^{\text {bcd }}$, Vivienne Baillie Gerritsen ${ }^{\text {bc }}$, and Vincent Zoete ${ }^{\text {ae* }}$
}

\begin{abstract}
The Drug Design Workshop initiative was put in place in 2015 and consists of a collection of educational tools especially developed to introduce computer-aided drug design to the general public and students of various levels. These presentations, hands-on sessions, physical material and on-line educational tools (http://www. drug-design-workshop.ch) have been used in a variety of settings including classrooms, universities, teacher training sessions and science fairs. The main goal is to inform an audience as broad as possible regarding the processes and challenges for the design, discovery and development of drugs. The present article describes what is presently available and the future direction for new activities currently under development.
\end{abstract}

Keywords: Drug discovery · Computer-assisted drug design · Education · General public · STEM



Antoine Daina holds a Federal Diploma of pharmacy and obtained a $\mathrm{PhD}$ in Pharmaceutical Sciences from the University of Geneva in 2006. He spent three years as a computational chemist at Syngenta Crop Protection, in the Chemistry Research Department and three years at the School of Pharmaceutical Sciences in Geneva as Senior Scientist/Lecturer.

${ }^{*}$ Correspondence: Prof. Dr. V. Zoete ${ }^{\mathrm{ae}}$

E-mail: vincent.zoete@sib.swiss


Bioinformatics

Bâtiment Génopode, Quartier Sorge,

$\mathrm{CH}-1015$ Lausanne

bOutreach Team, SIB Swiss Institute of Bioinformatics Bâtiment Génopode, Quartier Sorge,

$\mathrm{CH}-1015$ Lausanne

'Swiss-Prot Group, SIB Swiss Institute of Bioinformatics, CMU

1 rue Michel Servet, University of Geneva,

$\mathrm{CH}-1211$ Geneva 4

dTraining Group, SIB Swiss Institute of Bioinformatics Bâtiment Génopode, Quartier Sorge,

$\mathrm{CH}-1015$ Lausanne

eDepartment of Fundamental Oncology and Ludwig Institute of Cancer Research

University of Lausanne, Route de la Corniche 9A, $\mathrm{CH}-1066$ Epalinges

${ }^{\ddagger}$ A.D. and M.C.B. contributed equally to this work.
Antoine Daina joined the Molecular Modeling Group at SIB, Swiss Institute of Bioinformatics in 2012 and is in charge of developing, providing and applying novel methods for computer-assisted drug design. His main research themes include chemoinformatics and machine-learning models for physicochemistry, pharmacokinetics and ADME. Besides his academic teaching duties, he is involved in the development of educational tools for drug design targeting high-school students and the general public, in the context of the Drug Design Workshop project.

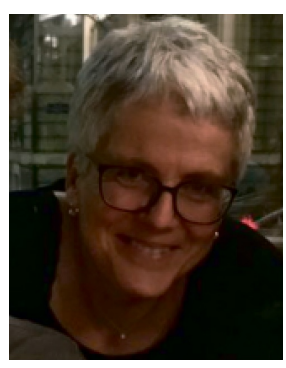

Marie-Claude Blatter has a $\mathrm{PhD}$ in Biochemistry. After several years in biomedical academic research, she is now responsible for the outreach activities at the SIB Swiss Institute of Bioinformatics. She coordinates events for classes (age 12-19) and for the public at large, takes an active part in them, and is responsible for training high-school teachers. She is also involved in biocuration and user support for the UniProtKB protein knowledgebase (www.uniprot.org) and is part of academic teaching program with special expertise in the fields of biological data \& knowledgebases.

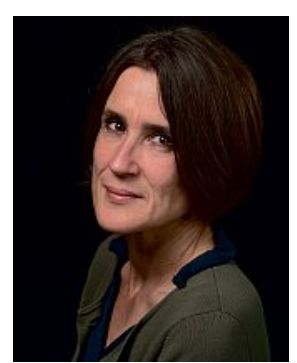

Vivienne Baillie Gerritsen holds a degree in Biology from the University of Geneva, and has been involved in popular science for many years. She is a science writer at SIB, and author of the 'Protein Spotlight' articles (www.proteinspotlight. org).

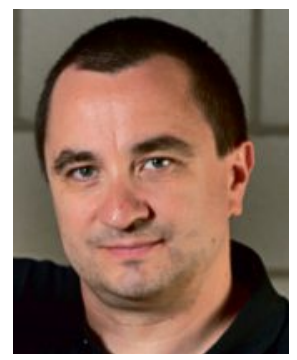

Following an engineer degree from the 'Ecole Nationale Supérieur de Chimie de Lille', a Master in Drug Design and a PhD in organic chemistry from the University of Lille in 1999, Vincent Zoete made a Postdoc in molecular modeling and drug design in Prof. Karplus' group in Strasbourg, and subsequently in Prof. Meuwly's group in Basel. He then joined the SIB Swiss Institute of Bioinformatics in 2004 where he was appointed as Associate Group Leader of the Molecular Modeling Group 
in 2011. He is also Assistant Professor in the Department of Fundamental Oncology at the University of Lausanne since 2017, and the Scientific Director of the Protein Modeling Facility of the same university. Together with Prof. Michielin, Vincent Zoete is in charge of the SwissDrugDesign project of the SIB, which consists of a collection of freely accessible web tools for computer-aided drug design, including SwissDock.ch, SwissParam.ch, SwissSidechain.ch, SwissTargetPrediction. ch, SwissBioisostere.ch, SwissSimilarity. ch and SwissADME.ch. He is also active in the field of education, notably in computer-aided drug design with the DrugDesign-workshop.ch project.

\section{Introduction - Motivations}

The chemical sciences have shown remarkable benefit for society. In particular, over the past century life expectancy and quality has been favorably impacted by the discovery, development and production of new pharmaceuticals. ${ }^{[1]}$

Furthermore, 'drugs' as a whole is a popular theme among school students, and drug discovery is itself an appreciated subject at university level. The success of a new treatment enables high visibility of the discipline for the public, which is informed by the generalist media about general concepts and global cost of 'making' drugs. ${ }^{[2]}$ However, the concrete challenges of drug discovery, and the research and technological tools that are available to achieve them, remain largely unknown to the general public and students. Here we focus on the approaches and methods which make use of computing resources - algorithms, databases and 3D-visualization - and are captured by the term Computer-Aided Drug Design (CADD). CADD aims at drawing rational hypotheses by performing chemi$\mathrm{cal} / \mathrm{structural}$ analyses in order to generate decision-making criteria which support the execution of drug-discovery processes the final aim being to improve efficiency in providing pharmaceutical agents for the patient. ${ }^{[3-5]}$

In this article, we describe our Drug Design Workshop, which consists of a collection of educational tools for CADD that are developed and maintained by us. These presentations, hands-on sessions, and on-line educational tools ( $h t t p: / / w w w$. drug-design-workshop.ch) are conducted in a variety of settings - from high-school classes and specialized educator trainings to science fairs - with great success. As an example, over 1'200 high-school students have taken part in our workshops reporting an overall high satisfaction level (5.0 out of 6.0 , on average). The positive feedbacks and many constructive discus- sions we have had stressed the need for a greater perception of 1) the drug development workflow and how computers deal with it (mainly among the younger participants); and 2) current challenges like personalized medicine or drug resistance (mainly for the well-informed public). This fostered the creation of additional material to broaden even further our targeted audience.

\section{Drug Design Workshop - What Exists}

Different educational materials - gathered under the general initiative 'Drug Design Workshop' - were conceived in a flexible way so as to be useable in a large variety of settings while targeting a diverse audience (pupils, high-school and university students as well as the general public). Developed activities (Table 1) share the same pedagogical objectives: i) to inform that most drugs are small synthetic molecules which interact with a protein in the body to trigger a therapeutic effect; ii) to reveal how time-consuming, complex and expensive drug discovery and development processes are; iii) to show that the process of discovering new drugs is a collective, interdisciplinary effort; iv) to explain how
CADD ('computers') supports the evaluation of key properties for a molecule to become an actual drug, e.g. affinity for the target, fate in the organism or possible side-effects.

\subsection{D-printed Protein and Drugs}

Although executed by computers, we felt that it was important for the audience to realize that CADD methods are based on tangible physical concepts. For instance, the fundamental notion of molecular recognition of a small drug molecule by a target protein can be modeled with rules related to classical mechanics (so-called molecular mechanics) including various potentials for internal and non-bonded energy terms (like Lennard-Jones or Coulomb potentials). The evaluation of how a small molecule binds to the macromolecular protein, and how strongly, are performed in silico by docking engines. These computer programs are able to predict the most probable conformation, orientation and position of the small molecule at the surface of the protein by optimizing the interactions between both molecular partners at the atomic level. The underlying 'lock-and-key' concept of docking is intuitive. As a means of introduction to the general public and students, a model of the protein (cyclooxygenase-1, COX1) targeted by nonsteroidal

Table 1. List of Drug Design Workshop activities described in the present article.

\begin{tabular}{|c|c|c|}
\hline Activity & Short description & Public \\
\hline $\begin{array}{l}\text { Educational } \\
\text { website }\end{array}$ & $\begin{array}{l}\text { www.drug-design-workshop.ch includes } \\
\text { three computer-assisted workshops, where } \\
\text { the objective is to design the 'best' possible } \\
\text { molecule to treat either inflammation, } \\
\text { melanoma or other cancers. }\end{array}$ & $\begin{array}{l}12 \text { years and } \\
\text { older }\end{array}$ \\
\hline $\begin{array}{l}\text { 3D-printed } \\
\text { protein and } \\
\text { drugs }\end{array}$ & $\begin{array}{l}\text { The COX protein and several anti- } \\
\text { inflammatory drugs, printed at the same scale, } \\
\text { allow for an intuitive hands-on illustration } \\
\text { of molecular docking and intermolecular } \\
\text { recognition. }\end{array}$ & $\begin{array}{l}8 \text { years and } \\
\text { older }\end{array}$ \\
\hline Wooden puzzle & $\begin{array}{l}\text { Young children can understand that it is } \\
\text { possible to design diverse molecules able to } \\
\text { fit into a protein cavity. }\end{array}$ & $5-8$ years \\
\hline $\begin{array}{l}\text { Deduction card } \\
\text { game }\end{array}$ & $\begin{array}{l}\text { This game illustrates the multi-objective } \\
\text { nature of drug discovery by searching the } \\
\text { molecule with all optimal properties (i.e. } \\
\text { non-toxic, water-soluble, well-absorbed, } \\
\text { stable, efficient in humans and selective to the } \\
\text { target). }\end{array}$ & $\begin{array}{l}8 \text { years and } \\
\text { older }\end{array}$ \\
\hline $\begin{array}{l}\text { Molecular } \\
\text { fingerprints } \\
\text { exercise }\end{array}$ & $\begin{array}{l}\text { The participant can reproduce with pen-and- } \\
\text { paper the process applied by a computer to } \\
\text { behold molecular structures and quantify the } \\
\text { similarity between them. It illustrates the } \\
\text { 'similarity principle' in drug design. }\end{array}$ & $\begin{array}{l}14 \text { years and } \\
\text { older }\end{array}$ \\
\hline
\end{tabular}


anti-inflammatory (NSAIDs) compounds was 3D-printed, as were the active ingredients of well-known anti-inflammatory drugs (e.g. ibuprofen, diclofenac, nimesulide). At first, the 3D-printed models are useful for beholding the relative sizes of the macromolecular protein and of the small molecule drugs. Manual docking can then be performed by opening the printed COX1 structure so that the binding site becomes accessible, and by positioning the printed drug molecule inside (such as ibuprofen as in Fig. 1). This approach is very approximate from a physics point of view because it only accounts for shape recognition and not for specific forces (like hydrophobic forces or those driven by hydrogen-bonding) nor molecular flexibility for instance. But we believe that this manual exercise helps to reveal clearly both the challenges of docking and the need for automation through computer algorithms. Such algorithms are able to increase dramatically the number of molecular structures which need to be handled while decreasing the extent of physical approximations.

\subsection{Web-Based Educational CADD}

A fully integrated Web interface was created to initiate beginners to the use of computers for designing molecules and evaluating their potentiality to become an actual drug. The website is freely available at www.drug-design-workshop.ch for the English version or at www.atelier-drugdesign.ch for the French version (translation in German is in progress), and can be considered as the cornerstone of the Drug Design Workshop. It involves introductory movies, documents, help pages and three workshops regarding pertinent therapeutic targets. Three proteins relevant to drug design were chosen: (i) both isoforms of cyclooxygenase (COX1 and COX2) with the aim of introducing the concept of specificity for a protein with regards to therapeutic versus unwanted effects of NSAIDs; (ii) B-Raf kinase for the notion of somatic mutation and targeted cancer therapy with game-changing drugs; like vemurafenib; and (iii) indoleamine 2,3-dioxygenase 1 (IDO1) as an example of current cancer immunotherapy targets involving research drug-candidates molecules. The biological context and usage of the workshops are extensively provided in dedicated Web pages and in a previous article. ${ }^{[6]}$ The main goal of these on-line workshops is to let the user enter the iterative cycle of designing and optimizing a molecule to make it a strong ligand for the protein target. This is achieved by hiding the technical complexity behind a simple and user-friendly interface, and by using enough approximations to keep the calculation time short (about two minutes or less) for the process to be truly interactive.
Briefly, as shown in Fig. 2 for the COX workshop, the input page includes pictures of the 3D structure of target proteins on the left, and boxes including 2D chemical structures of representative drugs on the right. A simple drag-and-drop of a given chemical structure on one protein gives di-

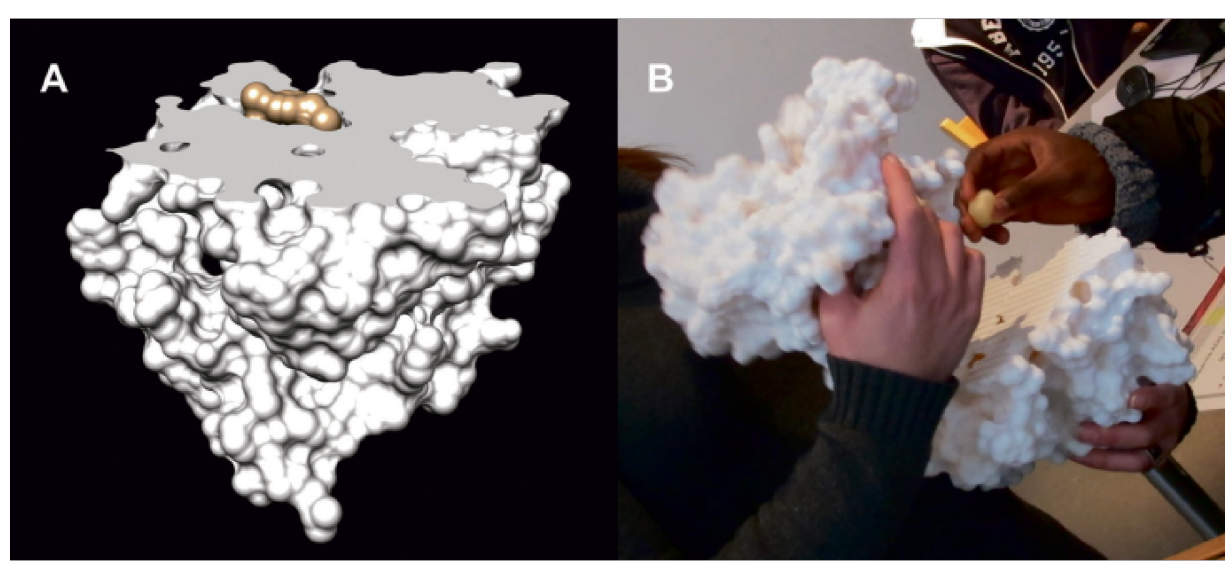

Fig. 1. The 3D model of cyclooxygenase-1 (COX1) and ibuprofen. (A) The computer model obtained from the crystal structure resolved by X-ray diffraction (pdb entry 1EQG) showing COX1 (molecular surface in white), the cutting plane and ibuprofen (molecular surface in beige) accommodated in the binding site. (B) The printed model employed in classroom for manual docking of ibuprofen in the opened COX1.

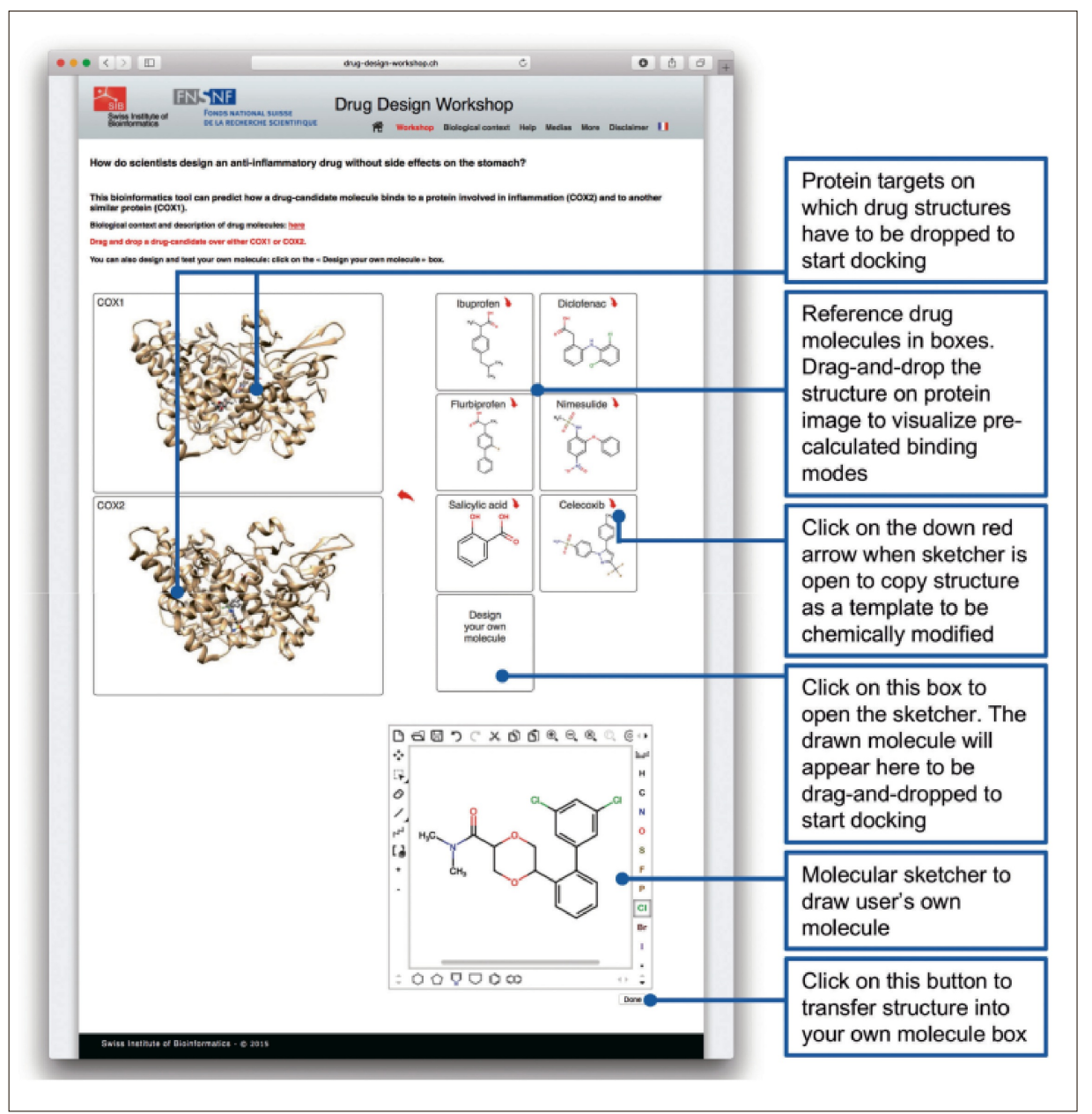

Fig. 2. An example of the Drug Design Workshop website input page, where technical complexity is hidden behind a user-friendly interface to allow simple drag-and-drop docking and easy sketching of molecules. Adapted from ref. [6]. rect access to a page including a 3D session of the pre-calculated docking pose. This session is interactive so that the user can zoom, rotate and translate the system with the mouse, to analyze visually the predicted binding mode. 
The user is invited to design his/her own tentative drug by clicking on the 'Design your own molecule' box of the input page. A molecular sketcher opens (MarvinJS version 6.1, 2013, www.chemaxon.com) to draw a chemical structure. For less experienced users, the sketcher can be filled with one of the pre-selected drugs, by clicking the down red arrow next to the structure. Any modifications can be then applied by using the sketcher toolbars. In our experience, even students without knowledge in organic chemistry are able to draw correct molecular structures with the help of the sketcher facilities.

By clicking the 'Done' button, the molecule appears in the corresponding box, and is available for docking with the same dragand-drop procedure as explained above.

All docking technical steps, i.e. preparation of ligand and protein as well as the docking calculation by AutoDock Vina, ${ }^{[7]}$ are transparent to the user. Although per- formed on a remote server, computation takes about 20 seconds to 2 minutes depending mainly on the size of binding site and ligand. Moreover, depending on the load of the server, the calculation can be queued. A panel informs the user on the progress of its run. In a standard setup, 15 users can perform basic steps on the website simultaneously without affecting interactivity.

Upon completion, one click displays the binding mode as an interactive 3D session in the result page, which also provides an evaluation of the binding strength (Fig. 3). For the sake of clarity, this score is the opposite of the binding free energy as predicted by Autodock Vina. ${ }^{[7]}$ This perception of "the larger the score, the better the ligand' and the relative scale for comparing with reference drugs is intuitive enough to strongly motivate the users to design 'better' molecules, and thus to enter effortlessly into the characteristic iterative optimization cycle inherent to CADD.

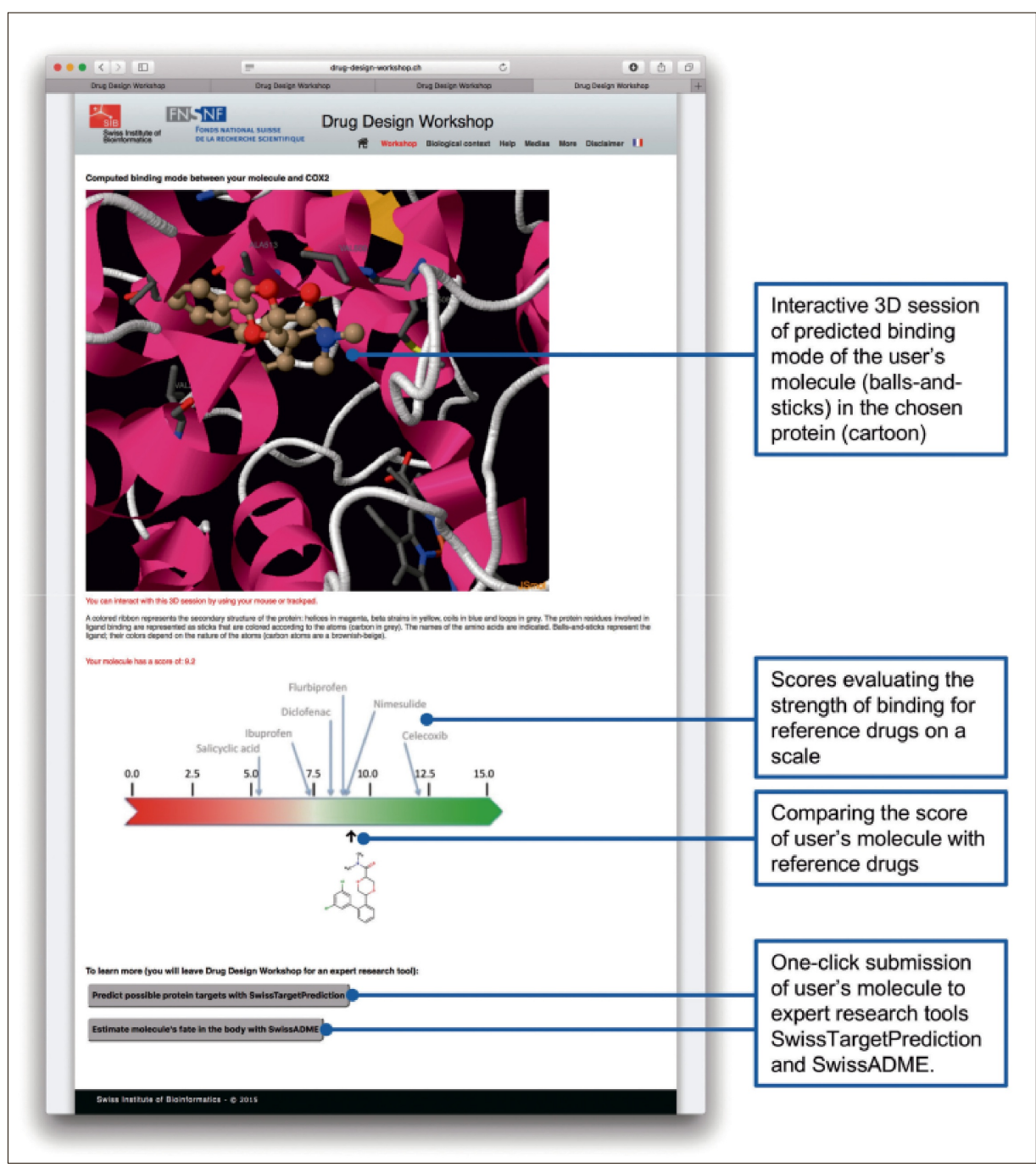

Fig. 3. Example of a result page for a molecule designed by a user of the Drug Design Workshop website and docked into COX2. The upper part is dedicated to the 3D interactive session to visualize the predicted binding mode; the middle part allows the comparison of the predicted potency (score) of the 'virtual' molecule with 'real' existing drugs; on the bottom of the page are two buttons that enable easy submission to research expert tools SwissTargetPrediction and SwissADME for further assessment of the potential of the user's molecule to become a drug. Adapted from ref. [6].

For more advanced participants, the possibility to access two professional research tools is given. By clicking on one of the two buttons at the bottom of the result page, the user's molecule is submitted either to SwissTargetPrediction ${ }^{[8]}$ to estimate its most probable protein targets or to SwissADME[9] for the calculation of physicochemical, pharmacokinetic and drug-likeness properties. The user can thus get a realistic 'feel' of the difference between research and educational tools. More important maybe is what the participant learns from these expert methods: i.e. that for a molecule to become a drug, it has not only to be recognized by its protein targets for potency, but it must also fulfil a number of criteria such as being non-toxic, showing few side-effects, baring the optimal properties to reach the protein target in the body, being quite rapidly eliminated, being synthesizable and stable, just to cite a few. With guidance from experts or well-trained educators, the central notion of CADD, which consists in a multiple objective iterative cyclic process, can be clearly understood.

This link to specialized methodologies that are systematically used in research settings was the first answer to demands that were formulated on feedbacks, and which asked for the workshop to be extended to a more informed audience. What is more, the biological context of the B-Raf workshop was enriched so as to touch the theme of personalized medicine. Focus is given on the importance of determining whether the melanoma cells of a given patient bears a specific mutation (V600E) or not. By sequencing its BRAF gene (Uniprot ID: P15056), the patient is defined eligible for treatment with drugs that inhibit this specific mutant, such as vemurafenib. In the case of melanoma cells that do not carry the V600E mutation of B-Raf, vemurafenib proved to be deleterious because it favored tumor growth. ${ }^{[10]}$ The blocking of melanoma cell proliferation by this medium constitutes a recent success story in the realm of drug design in cancer targeted therapy and personalized medicine. A movie exemplifying these aspects at molecular level is available on the website. Other workshops in the context of individualized therapy and drug resistance shall be implemented in the near future.

\section{Broadening the Audience to a Younger Public}

The other major demands were to extend the targeted audience to younger participants, either in the classroom or in scientific events. Following this, various 
activities were developed to depict and exemplify key notions of CADD especially for youngsters.

\subsection{Wooden Puzzle}

The activities regarding molecular recognition and iterative cyclic optimization described above, i.e. the 3D-printed models for the manual docking NSAIDs (Fig. 1) and the online workshop for virtual docking (Figs 2 and 3 ) have proven to be excellent tools for introducing important concepts of CADD. However, they turned out to be too advanced for the younger participants. Therefore, we designed a simple puzzle in wood, which enables children from age 5 onwards to build and dock small molecules into a 2D protein, by filling the binding site cavities with puzzle pieces representing molecular fragments (Fig. 4). This tool introduces effortlessly the notion of shape complementarity and scaffold hopping since different pieces can fill the same pocket. Younger children are able to understand that diverse molecules can achieve the 'lock-and-key' objective, while more advanced participants can calculate how many well-fitting molecules can be generated.

\subsection{Deduction Card Game}

Finding a molecule with a shape that fits into the protein binding site is certainly needed but not sufficient to propose a drug candidate. Other aspects such as the molecule's fate in the organism, possible side effects and toxicity must be investigated. To illustrate this important aspect of CADD, we have developed a deduction game, whose goal is to find among six cards with one molecule on each, the best drug candidate (Fig. 5). The best candidate is the molecule that displays all optimal properties, i.e. non-toxic, water-soluble, well-absorbed, stable, efficient in humans and selective to the target. All other five possible cards show molecules with at least one sub-optimal property. Each property is linked to a specific fragment in the molecule, and this link has to be deduced from a pool of cards displaying molecules with sub-optimal properties (for an example, refer to Fig. 5A). The number of cards can vary so as to adapt the difficulty to the level of the participants (from the age of 8). Once the participant has attributed a given sub-optimal property to a molecular fragment, he/she can identify, among the six molecules mentioned above, the one that does not show any of the problematic fragments. Of note, participants do not need to master concepts in organic chemistry to be able to do this exercise: understanding is straightforward when considering molecules as images with a common part (the scaffold) and differences (the molecular fragments). The tutors can then introduce



Fig. 4. Wooden puzzle for young children to perform manual docking $(A)$ first outline; and $(B)$ welladvanced prototypes tested in a science fair setting. the concept of chemical structures if required, or useful.

\subsection{Molecular Fingerprints Exercise}

Apart from methodologies using information about the structure of the protein targets ('structure-based'), other CADD approaches depend on the knowledge of small molecules with desired activities. The rational of such 'ligand-based' approaches is founded on the similarity principle, which assumes that similar molecules are prone to exhibit similar biological activities. ${ }^{[11,12]}$ This central concept can, for instance, be used to perform virtual screening: i.e. looking into a library of (commercially) available molecules that are similar to an active one, and which should be tested experimentally in priority. Computers are needed to be able to rapidly quantify molecular similarities between the millions of molecules included in chemical libraries nowadays. The way a computer performs this task for a couple of molecules provides an excellent opportunity for STEM (Science, Technology, Engineering and Mathematics) teaching, ${ }^{[13]}$ by making a direct link between mathematics, informatics, chemistry and drug design. We have designed a pen-and-paper exercise so that participants can actively experience how to translate an intuitive qualitative estimation of molecular similarity - like a hu- man can do - into a quantitative evaluation by simple maths and an algorithm applicable through a computer program. With this in mind, we used a technique called 'molecular fingerprints' that translates a chemical structure into a bit string of 0 and 1, which is the actual regular object of computer calculations. ${ }^{[14,15]}$ A simplified protocol is given to the participant to create fingerprints and manipulate them for calculating molecular similarity 'by hand' (Fig. 6). For a series of drug molecules, with the help of a transparent small ruler on which chemical fragments are drawn, participants note the presence or absence of fragments by writing in the related bit of an empty vector either ' 1 ' (fragment is found in the molecule), or ' 0 ' (not found). The so completed vectors of each molecule are then compared two-by-two by calculating the Tanimoto coefficient (TC). ${ }^{[16]} \mathrm{TC}$ is obtained by dividing the number of times ' 1 ' is found in the same column of both vectors by the number of times ' 1 ' is found in at least one of the two vectors. The notion of vector does not necessarily need to be introduced, since it simply (and naturally) translates into a table in this exercise. Finally, by confronting TC between known therapeutic classes of molecules (here antiinflammatory versus anti-cancer drugs) the comes concrete. application of the similarity principle be-
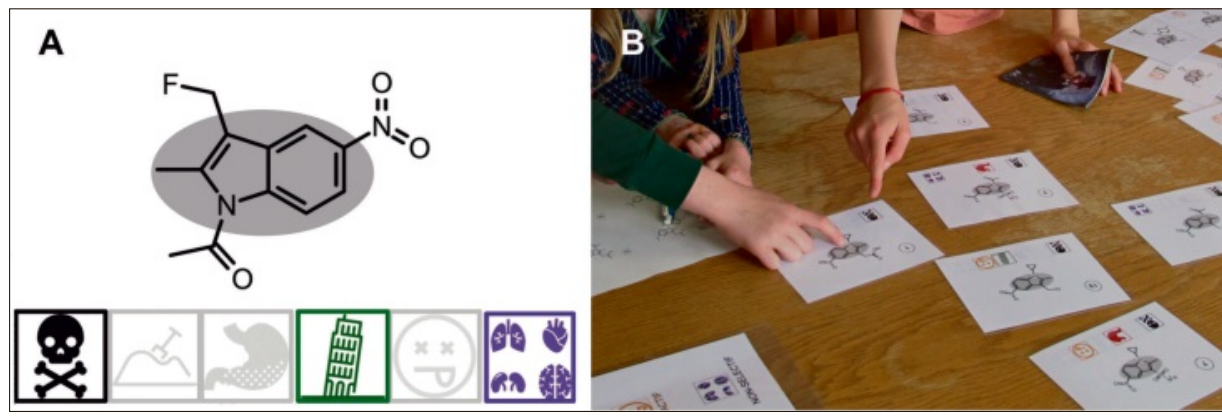

Fig. 5. Deduction card game dedicated for multi-objective optimization. (A) Design of a card used for deduction: in this example, the molecule, three sub-optimal properties, i.e. toxicity (black skull), instability (green Pisa tower) and non-specificity (purple organs), are to be linked with the three fragments of the molecule; and $(B)$ prototypes of cards tested during a science fair. 
For full practicality, reusable physical supports including all information (at the moment available only in French as in Fig. 6C) were produced for science fairs and classrooms. In our experience, the general public and children from 14 years old are able to do this exercise and understand the concrete application of mathematics and engineering to the field of chemistry. An online version of the exercise is planned to be added to our Drug Design website.

\section{Outcome and Outlook}

The Drug Design Workshop activities are all independent from one another and have different pedagogical objectives. However, they can also be presented in a logical manner. Participants can do one or several activities depending on their availability and level of understanding. This allows us to target a wide audience, from the youngest (aged 5-12), secondary I (12-15) and secondary II (15-19) students to school teachers, and even Bachelor or Master students. The general public is reached in science fairs or other events.

Since 2015, more than 1'200 high school students have attended the workshops and, with our guidance, have designed and evaluated molecules for their potential to become a drug. Note that our website has also been designed simple enough so that one person can use it without the need of tutor guidance. Since 2015, it has received around 10'000 unique visitors
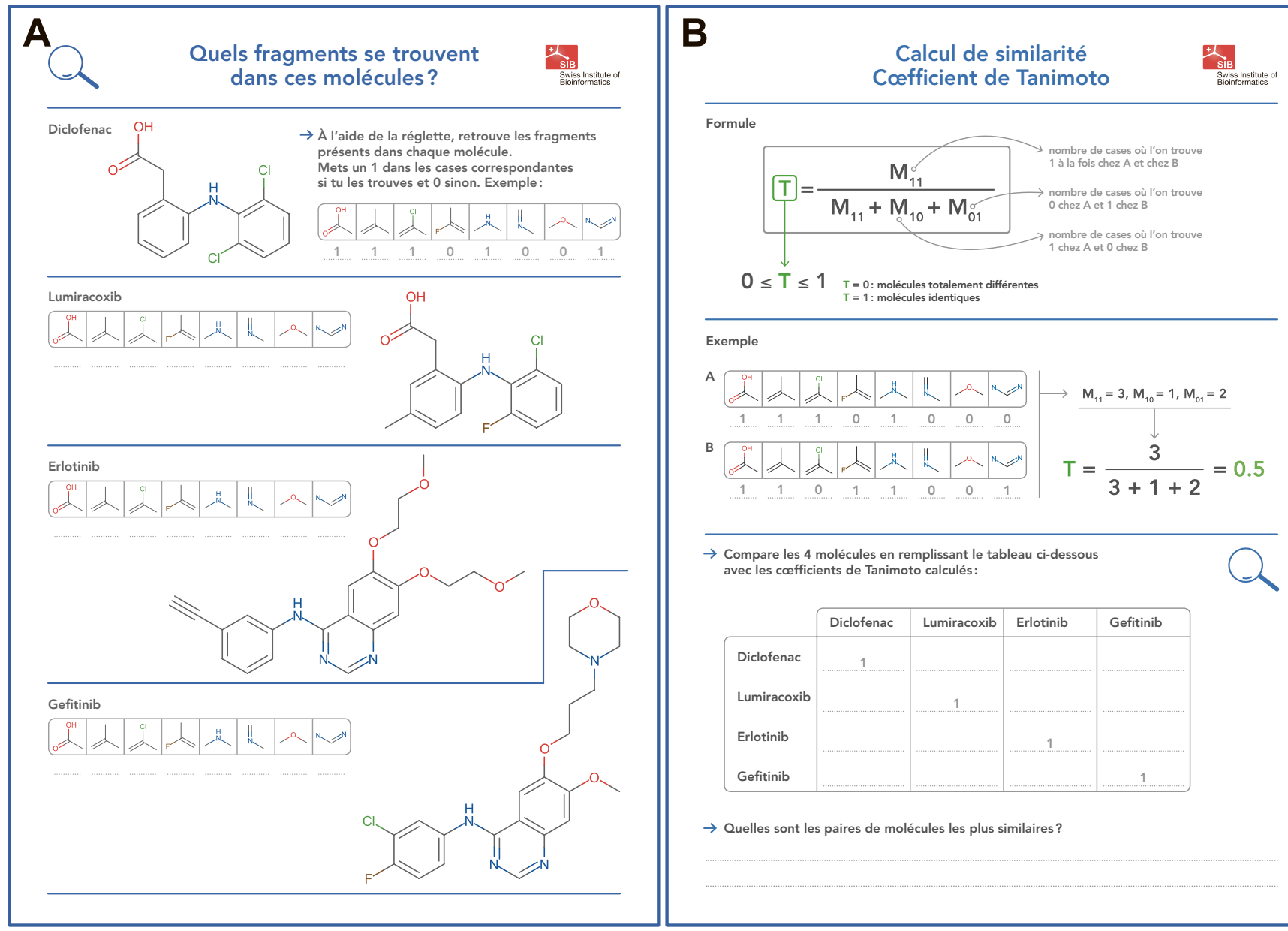

$\rightarrow$ Quelles sont les paires de molécules les plus similaires?

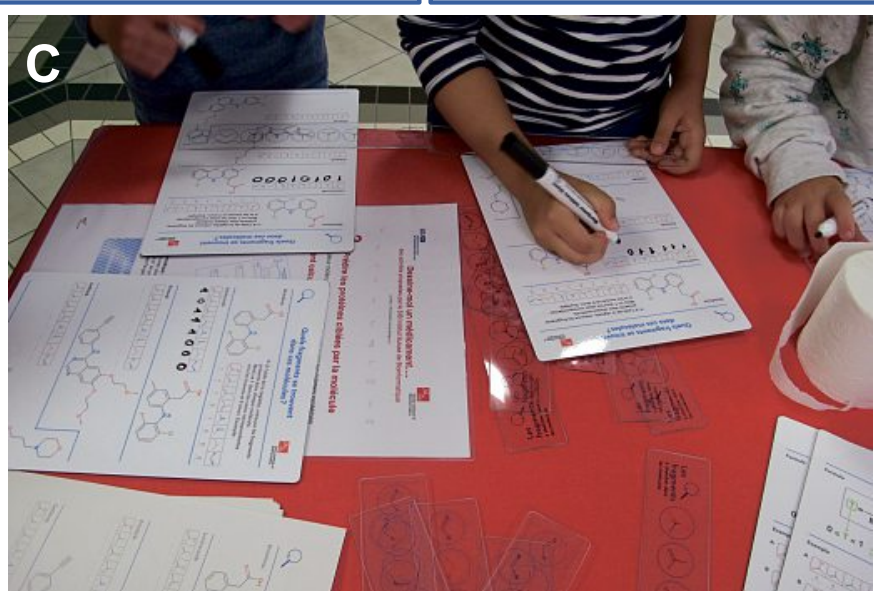

Fig. 6. STEM exercise on Molecular Fingerprints. (A) Design of the tablet's first page, which allows to complete the binary bit strings as a computer would translate molecular structures; (B) Design of the tablet's second page, giving all information on how to quantify chemical similarity between molecules; and (C) young students employing the reusable supports and the small transparent rulers to calculate the molecular similarity between pairs of drugs. 
who have opened about $15^{\prime} 000$ sessions. $27 \%$ of the session were from Switzerland $19 \%$ from the United States and the rest is spread worldwide.

According to the comments we have received, the participants appreciated the concreteness and realism of the activities. It allowed them to use bioinformatics and professional tools in a real scientific process. Participants also liked the given overview of how scientists work nowadays. They appreciated observing and experiencing the various steps and parameters required for the design of a new drug. Last but not least, our activities were appreciated because they differ from the usual classroom activities, mainly thanks to their interdisciplinary nature (chemistry, biology, mathematics, informatics and medicine). This highlights the usefulness of the Drug Design Workshop for STEM teaching which remains challenging, especially regarding high-school teacher training.

Our workshops have also proved to be practical in introducing drug design concepts to the general public in scientific popular events. About 2'500 visitors, including pupils from the age of 8 , were introduced to CADD during several science fairs and university open house days. They appreciated being offered the opportunity to discover an area of science related to drugs and medicine that concerns each and every one, and which is often the subject of debate. The proposed activities rendered opportunities to discuss drug discovery and development pipeline, duration, costs and challenges.

We consider that it is of paramount importance to stay as close as possible to the latest discoveries in biomedical research and Life Sciences. In that respect, we continue to develop additional activities in the context of the Drug Design Workshop to introduce the most recent concepts in therapeutics, including personalized medicine and drug resistance. Beside information of the public at large, one objective is to stimulate and frame scientific curiosity and to help students to discover new areas of research, with the hope of facilitating their career choice.

\section{Associated content}

Short movies and documents introducing the notions mentioned in the present article are available under the CC-BY-ND-NC license on the Drug Design Workshop web site at http:// www.drug-design-workshop.ch for the English version and http://www.atelier-drug-design.ch for the French version.

\section{Acknowledgments}

This work was supported by SIB, Swiss Institute of Bioinformatics and by the Swiss National Science Foundation through Agora grants CRAGP3_151515 (to Prof. Olivier Michielin and V.Z.) and CRAGP3_171653 (to V.Z. and A.D.). The author would like to thank colleagues at the SIB, Swiss Institute of Bioinformatics, who have been involved in conducting or elaborating parts of the Drug Design Workshop, in particular Drs. Diana Marek and Patricia Palagi. Profs. Olivier Michielin and Ioannis Xenarios are also gratefully acknowledged. Noémi Savary, studio KO, Yverdon-les-Bains, Switzerland was extensively involved in the design and making of movies and pen-and-paper supports. Caroline Emmelot at Decologic, Vevey, Switzerland produced the wooden puzzle prototypes. ChemAxon is acknowledge for the academic license agreement. Molecular docking is performed by AutodockVina and the 3D session embedded within the web pages are JSmol applets.

Received: September 29, 2017

[1] F. R. Lichtenberg, Health Policy and Technology 2014, 3, 36 .

[2] T. R. Helgren, T. J. Hagen, J. Chem. Educ 2017, 94, 345

[3] V. Zoete, A. Grosdidier, O. Michielin, J. Cell. Mol. Med. 2009, 13, 238

[4] G. Schneider, Chimia 2012, 66, 120.

[5] W. L. Jorgensen, Acc. Chem. Res. 2009, 42, 724.

[6] A. Daina, M.-C. Blatter, V. Baillie Gerritsen, P. M. Palagi, D. Marek, I. Xenarios, T. Schwede, O. Michielin, V. Zoete, J. Chem. Educ. 2017 , 94, 335 .

[7] O. Trott, A. J. Olson, J. Comput. Chem. 2010, 31,455 .

[8] D. Gfeller, A. Grosdidier, M. Wirth, A. Daina, O. Michielin, V. Zoete, Nucleic Acids Res. 2014, 42, W32.

[9] A. Daina, O. Michielin, V. Zoete, Sci. Rep. 2017, 7, 42717.

[10] G. Bollag, J. Tsai, J. Zhang, C. Zhang, P. Ibrahim, K. Nolop, P. Hirth, Nat. Rev. Drug Discov. 2012, 11, 873 .

[11] Y. C. Martin, J. L. Kofron, L. M. Traphagen, J. Med. Chem. 2002, 45, 4350

[12] A. Bender, R. C. Glen, Org. Biomol. Chem. 2004, 2, 3204

[13] S. Freeman, S. L. Eddy, M. McDonough, M. K. Smith, N. Okoroafor, H. Jordt, M. P. Wenderoth, Proc. Natl. Acad. Sci. U.S.A. 2014, 111, 8410.

[14] I. Muegge, P. Mukherjee, Exp. Opin. Drug Discov. 2016, 11, 137.

[15] A. Cereto-Massaguà, M. J. Ojeda, C. Valls, M. Mulero, S. Garcia-Vallvé, G. Pujadas, Methods $\mathbf{2 0 1 5}, 71,58$.

[16] P. Willett, J. M. Barnard, G. M. Downs, J. Chem. Inf. Model. 1998, 38, 983. 